\title{
Self-Organizing Mobility Control in Wireless Sensor and Actor Networks Based on Virtual Electrostatic Interactions
}

\author{
Bartłomiej Płaczek $^{1}$ (D) Marcin Bernas $^{1}$ (D)
}

Published online: 27 September 2016

(C) The Author(s) 2016. This article is published with open access at Springerlink.com

\begin{abstract}
This paper introduces a new mobility control method for surveillance applications of wireless sensor and actor networks. The proposed method is based on virtual electrostatic forces which act on actors to coordinate their movements. The definition of virtual forces is inspired by Coulomb's law from physics. Each actor calculates the virtual forces independently based on known locations of its neighbours and predetermined borders of the monitored area. The virtual forces generate movements of actors. This approach enables effective deployment of actors at the initial stage as well as adaptation of actors' placement to variable conditions during execution of the surveillance task without the need of any central controller. Effectiveness of the introduced method was experimentally evaluated in a simulation environment. The experimental results demonstrate that the proposed method enables more effective organization of the actors' mobility than state-of-the-art approaches.
\end{abstract}

Keywords Wireless sensor and actor networks · Mobility control · Virtual forces · Surveillance applications

\section{Introduction}

Wireless sensor and actor networks (WSANs) are composed of sensor nodes and actors that are coordinated via wireless communications to perform distributed sensing and acting tasks. In WSANs, sensor nodes collect information about the physical world, while actors use the collected information to take decisions and perform appropriate actions upon the environment $[1,2]$. The sensor nodes are usually small devices with limited energy resources, computation

Bartłomiej Płaczek

placzek.bartlomiej@gmail.com

Marcin Bernas

marcin.bernas@gmail.com

1 Institute of Computer Science, University of Silesia, Będzińska 39, 41-200 Sosnowiec, Poland 
capabilities and short wireless communication range. In contrast, the actors are equipped with better processing capability, stronger transmission powers and longer battery life. The number of actors in WSAN is significantly lower than the number of sensor nodes [3, 4].

The WSANs technology has enabled new surveillance applications, where sensor nodes detect targets of interest over a large area. The information collected by sensor nodes allows mobile actors to achieve surveillance goals such as target tracking and capture. Several examples of the WSAN-based surveillance applications can be found in the related literature, including land mine destruction [5], chasing of intruders [6], forest fires extinguishing [7], and industrial systems [8]. Such applications require appropriate algorithm to control the mobility of actors. The mobility control algorithm should enable a self-organizing deployment of actors in the monitored area and ensure a fast access of actors to detected targets.

This paper introduces a mobility control approach for the surveillance applications of WSAN, where the sensor nodes detect stationary targets and report their positions to actors. On the basis of the received information, each actor selects the nearest target and moves toward it. A target is eliminated if an actor is close to the location in which the target was detected. The targets may correspond to intruders, landmines, enemy units, fires, etc.

The proposed approach is based on virtual electrostatic forces which act on actors to coordinate their movements. The definition of virtual forces is inspired by Coulomb's law from physics. Each actor calculates the virtual forces independently based on known locations of its neighbours and predetermined borders of the monitored area. The virtual forces generate movements of actors. This approach enables effective deployment of actors at the initial stage as well as adaptation of actors' placement to variable conditions during execution of the surveillance task without the need of any central controller. Initially, all actors have equal virtual electrostatic charges that result in uniform spatial distribution of actors in the monitored area. During execution of the target chasing task, the electrostatic charge is updated as a function of recent actor's workload. Consequently, a higher spatial density of actors is obtained in regions where the targets are detected more frequently. Moreover, in case of actor failure, the proposed method allows the remaining active actors to be appropriately repositioned. Main advantages of the proposed self-organizing mobility control are robustness, scalability, flexibility, adaptivity, and low computational cost.

Effectiveness of the introduced approach was experimentally evaluated in a simulation environment. The experimental results demonstrate that this approach achieves a good performance in terms of average time from target detection to elimination and total distance covered by an actor during target chasing.

The paper is organized as follows. Section 2 includes review of related research and describes main contribution of this paper. In Sect. 3, the method of self-organizing actors' mobility control is introduced in details. Section 4 contains results of the experimental evaluation. The performance of the proposed method is compared against results obtained for state-of-the-art approaches. Finally, in Sect. 5, conclusions are presented and some future research directions are outlined.

\section{Related Works and Contribution}

\subsection{Control of Actors' Mobility}

In the literature, a number of works can be found that are related to the mobility control of WSANs, where actors have to be moved toward events or targets detected by sensor nodes. Most of these works deal with the issues of sensor-actor communication and coordination 
of the actors' mobility after target (or event) detection. However, little research is focused on controlling the mobile actors before they receive the information about detected target form sensor nodes. More research is necessary to provide effective methods for management of the actors' location prior to target detection. This task is crucial for the considered surveillance applications because if an actor is available close to the location where a target is detected then the target can be eliminated in a short time. Moreover, if the locations of the actors are close to the locations of the detected targets then a lower number of sensor nodes is involved in relaying data to the actors and their energy consumption is reduced [9].

The most popular approach in the literature involves random deployment of the actors that remain static and move only when a target is reported [10-12]. In [13] the deployment of actors was managed by using an algorithm, which generates a connected pseudo-random unit graphs. The aim of this algorithm is to distribute the actors uniformly in the monitored area, while maintaining their connectivity. According to another popular method, actors walk randomly in the monitored area until they receive target reports from sensor nodes [14]. In [15] default locations of actors were determined to ensure that the communication ranges of the available actors cover the entire monitored area. The default locations are arranged in a square grid. An actor, which has not received information about detected target from sensor nodes moves toward its default location.

Ota et al. [14] have proposed an approach, which allows the actors to move towards predicted locations of events. In order to predict the location of an event, actors collect sensor readings along their way. Thus, additional energy is consumed by sensor nodes for periodic data transmission to actors that are moving in their vicinity. The prediction is based on maximum likelihood estimation. This approach is useful only for predictable events, such as occurrence of fire, which can be predicted based on temperature measurements. In case of targets occurring at random locations, that cannot be accurately predicted from sensor readings, the practical usefulness of this method is limited.

\subsection{Virtual Forces}

This paper introduces a method for the actors' mobility control in WASN by means of virtual forces. The concept of virtual forces was originally formulated in [16] as an element of the artificial physics framework for multi-agent systems. In this framework, the virtual forces were used to drive a multi-agent system toward a desired configuration that minimizes overall potential energy of the system. Under such assumptions, the system acts as a molecular dynamics simulation. Spears et al. [16] have suggested two potential advantages of this approach. First, a complex control is achievable through simple local interactions based on the virtual forces. Second, the approach scale well to larger sets of controlled entities.

In the literature, several methods were proposed that utilize virtual forces for sensor node deployment and mobility management in wireless sensor networks (WSNs). Applications of these methods have demonstrated capabilities of self-organization, fault-tolerance, and self-repair [17, 18].

The deployment problem of mobile WSNs was considered in [19]. The goal of this problem was to find locations and movements of sensor nodes that result in maximum coverage and to form a uniformly distributed WSN in minimum time, with minimum energy consumption. In order to define the movement of sensor nodes during the deployment process, a virtual repulsive force was used, which depends on distance between sensor nodes and on local node density. 
The topology management techniques based on virtual forces enable handling of node failures in WSNs. These techniques utilize different definitions of repelling forces [20] and diffusion forces among sensor nodes [21]. A model of electrostatic interactions based on Coulomb's law was applied in [22] to restore connectivity in fragmented WSN after simultaneous failure of multiple nodes. In this scheme, the virtual forces spread sensor nodes and move them toward centre of the deployment area.

A mobile wireless network of homogenous sensor-actuator nodes was considered in [23]. The virtual forces were utilized in that study to form a connected network with roughly the same node density and relocate the nodes around a target location when they have to react to an event. It was assumed that the task of the sensor-actuator nodes is to detect fires in a forest area and extinguish them. Upon occurrence of a fire, the nodes surround the event area and track the movement of the fire front, while staying at a safe distance from which they can extinguish the fire without being damaged. The node density is increased in proximity of the fire front to stop its expansion. Such node behaviour was obtained by using virtual forces of three types. Exchange forces are established between pairs of nodes which are sufficiently close to each other. Potential forces attract/repel nodes toward/from the fire. Friction forces have been introduced to stop the nodes around an equilibrium configuration defined by potential and exchange forces.

Akkaya and Janapala [24] have presented an algorithm for actors' deployment in WSANs, which increases the connected actor coverage through relocation of actors based on repelling forces from neighbouring actors and from boundary sensor nodes. The main idea behind that approach is to apply repelling forces among neighbouring actors, similar to intermolecular forces in physics, in order to spread them in the deployment area. Furthermore, sensors on the boundaries of the deployment area also impose forces to actors so that they will not move outside the monitored region. The forces are applied in steps until convergence, when there is no further movement in the network. This algorithm does not take into account the necessity of actors' relocation toward detected events or targets.

\subsection{Contribution}

In this paper a new method is proposed for self-organizing control of actors' mobility in WSANs. The proposed method is based on virtual electrostatic interactions inspired by Coulomb's law from physics, which describes forces acting between electrically charged particles. The objective of this method is to enable effective relocation of actors that have to reach targets detected by sensor nodes in minimum time. Movement of an actor is controlled by the virtual forces if there is no target reported to this particular actor. When the actor receives a message about detected target from sensor nodes then it ignores the virtual forces and moves directly toward the target to eliminate it as fast as possible.

In this scheme, the virtual forces allow the actors' deployment to be dynamically adapted to local probability of target detection. The adaptation ability was obtained by introducing variable virtual electrostatic charges of actors. According to the proposed approach, the virtual charge of an actor depends on recent actor's workload. Exponential forgetting algorithm is applied to estimate the workload of each actor in some recent time period. The proposed definition of virtual electrostatic charges leads to reduced distances between actors in regions, where targets are detected more frequently. Thus, the resulting spatial distribution of actors is more effective than the uniform one, and the targets can be eliminated in shorter time.

It should be also noted that in contrast with the state-of-the-art methods, the proposed method does not require any additional energy-expensive communication with sensor 
nodes. The virtual forces and charges are determined on the basis of data transmitted only between neighbouring actors. They enable appropriate relocation of actors prior to target detection. Moreover, this method is effective also in case when the locations of targets cannot be accurately predicted.

\section{Proposed Method}

This section introduces details of the proposed method which enables self-organizing coordination of actors' mobility for effective target elimination in a monitored area. As it was already mentioned, the proposed method was inspired by Coulomb's law from physics that describes electrostatic interaction between electrically charged particles.

In Fig. 1 the Coulomb's law is illustrated for a pair of virtual electrostatic charges $\left(q_{1}\right.$, $q_{2}$ ) assigned to two actors. In the presented approach the charges of actors have the same sign, thus the electrostatic forces between them are repulsive. The electrostatic force acts along the straight line joining the actors and its magnitude is given by the following formula:

$$
\left|\mathbf{F}_{1,2}\right|=\left|\mathbf{F}_{2,1}\right|=k \cdot \frac{q_{1} \cdot q_{2}}{d^{2}},
$$

where $d$ denotes the distance between actors, and $k$ is a proportionality constant. For the sake of simplicity, it is further assumed that $k=1$.

In addition to the forces acting between actors, the proposed method also takes into account forces between actors and borders of the monitored area. Figure 2 shows an example of all repulsive forces that influence a single actor. For each actor, the repulsive forces of four borders (south, west, north, and east) are considered. These forces are determined along vertical and horizontal coordinates.

Resultant force for $i$-th actor $\left(\mathbf{F}_{i}\right)$ is calculated as a vector sum of all the repulsive forces acting on it:

$$
\mathbf{F}_{i}=\sum_{j \in A_{i}} \mathbf{F}_{i, j}+\mathbf{F}_{i, \mathrm{~N}}+\mathbf{F}_{i, \mathrm{E}}+\mathbf{F}_{i, \mathrm{~S}}+\mathbf{F}_{i, \mathrm{~W}}
$$

where $A_{i}$ is a set of indices $(j)$ of neighbouring actors, $\mathbf{F}_{i, j}$ denotes repulsive force of actor $j$ acting on actor $i, \mathbf{F}_{i, \mathrm{~S}}, \mathbf{F}_{i, \mathrm{~W}}, \mathbf{F}_{i, \mathrm{~N}}, \mathbf{F}_{i, \mathrm{E}}$ are the repulsive forces of sout, west, north, and east borders respectively.

According to the proposed approach, each actor periodically broadcasts its location to other actors in a communication range. Thus, during calculation of the resultant force $\mathbf{F}_{i}$ only those actors are considered that are in communication range of actor $i$. It means that set $A_{i}$ can be determined as follows:

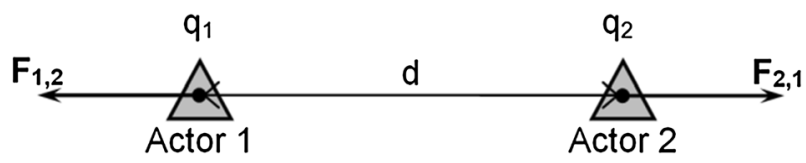

Fig. 1 Coulomb's law applied to actors with virtual electrostatic charges 


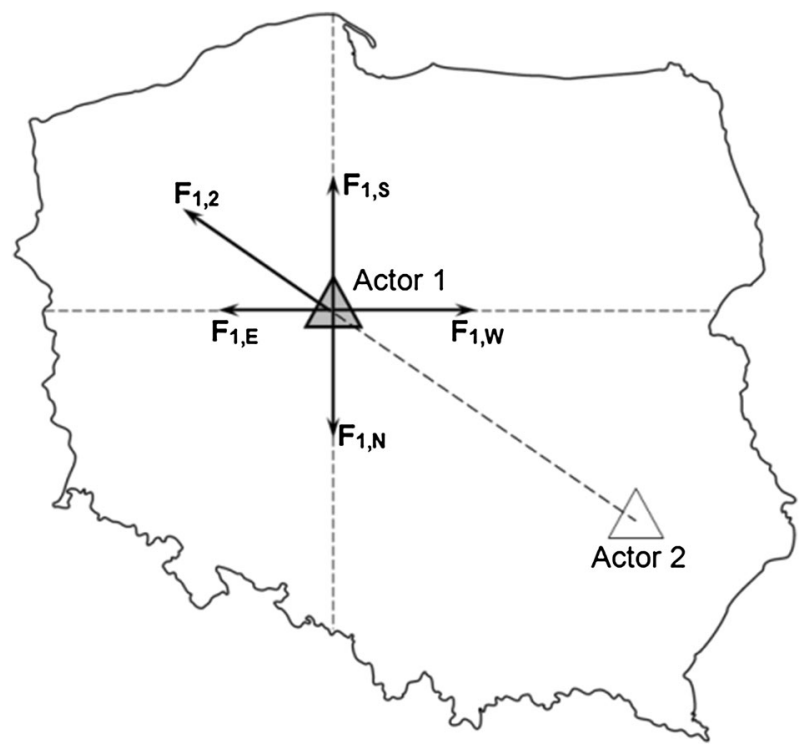

Fig. 2 Virtual forces acting on actor 1: repulsive force of actor $2\left(\mathrm{~F}_{1,2}\right)$ and repulsive forces of borders $\left(\mathrm{F}_{1, \mathrm{~S}}\right.$, $\left.\mathrm{F}_{1, \mathrm{~W}}, \mathrm{~F}_{1, \mathrm{~N}}, \mathrm{~F}_{1, \mathrm{E}}\right)$

$$
A_{i}=\left\{j: d\left(\mathbf{L}_{i}, \mathbf{L}_{j}\right)<r_{\mathrm{COM}}\right\},
$$

where $\mathbf{L}_{i}, \mathbf{L}_{j}$ are actual locations of $i$-th and $j$-th actor, $r_{\mathrm{COM}}$ denotes communication range of actors, and $d(\cdot)$ is the Euclidean distance between locations.

Movement of an actor depends on the resultant force. However, in order to avoid unnecessary moves of actors due to some minor force variations, it was assumed that the resultant force influences a destination location of the actor $\left(\mathbf{L}_{i}^{+}\right)$instead of the actual location $\mathbf{L}_{i}$. Actor moves toward the destination location, i.e., changes its actual location, only when the distance between actual location and the destination location $d\left(\mathbf{L}_{i}, \mathbf{L}_{i}^{+}\right)$is above its acting range $r_{\mathrm{ACT}}$. The destination locations are taken into account during calculations of the repulsive forces of actors and borders.

In some specific circumstances, the application of Coulomb's law can lead to metastable states with ineffective deployment of actors over the monitored area. An example of such metastable state is presented in Fig. 3, where all actors get stuck at half height of the square area as they are repelled by the north and south borders with forces of equal magnitudes. In order to eliminate the suboptimal metastable states, fluctuations of the repulsive forces are modelled by adding a random component to the Coulomb's formula.

Summarizing the above assumptions, the magnitude of repulsive force of actor $j$ is calculated for actor $i$ by using the following equation:

$$
\left|\mathbf{F}_{i, j}\right|=\frac{q_{i} \cdot q_{j}}{d\left(\mathbf{L}_{i}^{+}, \mathbf{L}_{j}^{+}\right)^{2}}+\sigma \cdot N_{i, j}(0,1),
$$

where $\sigma$ is a force fluctuation factor and $N_{i, j}(0,1)$ denotes an independent random sample from the standard normal distribution. 


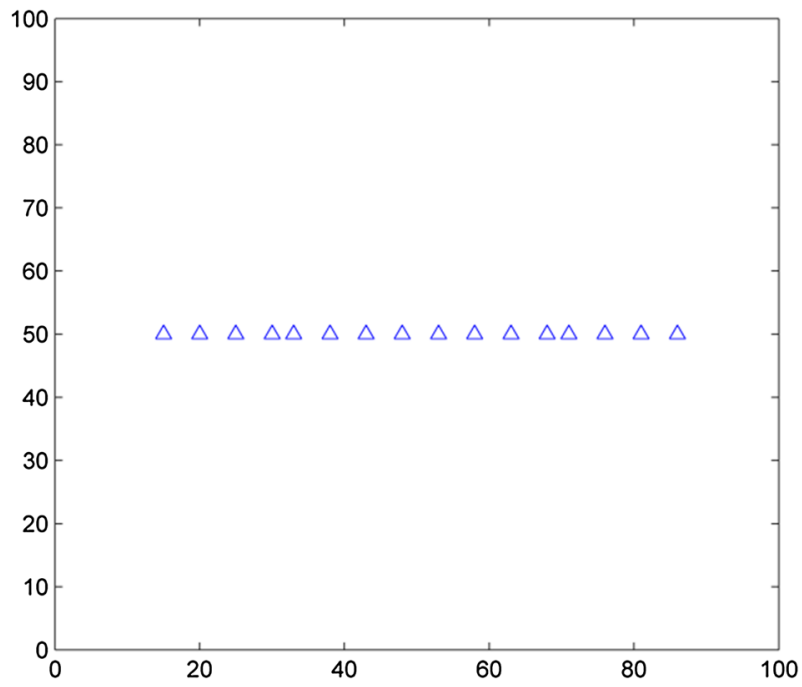

Fig. 3 Suboptimal metastable state: actor locations (triangles) in square area, no target detected

Magnitudes of the repulsive forces between borders of the monitored area and $i$-th actor are calculated in a similar way. For instance, when the repulsive force of south border is considered then the formula takes the following form:

$$
\left|\mathbf{F}_{i, \mathrm{~S}}\right|=\frac{q_{i} \cdot q_{\mathrm{B}}}{d\left(\mathbf{L}_{i}^{+}, \mathbf{L}_{i}^{\mathrm{S}}\right)^{2}}+\sigma \cdot N_{i, \mathrm{~S}}(0,1),
$$

where $q_{B}$ denotes a constant virtual charge of the borders, $\mathbf{L}_{i}^{\mathbf{S}}$ is a location at south border which corresponds to the horizontal coordinate $x_{i}^{+}$of the actor's destination location $\mathbf{L}_{i}^{+}=\left(x_{i}^{+}, y_{i}^{+}\right)$:

$$
\mathbf{L}_{i}^{\mathrm{S}}=\left(x_{i}^{+}, f_{\mathrm{S}}\left(x_{i}^{+}, y_{i}^{+}\right)\right),
$$

and $f_{\mathrm{S}}\left(x_{i}^{+}, y_{i}^{+}\right)$is a function defining south border of the monitored area.

Magnitudes of the repulsive forces from the remaining three borders, i.e., $\left|\mathbf{F}_{i, \mathrm{~W}}\right|,\left|\mathbf{F}_{i, \mathrm{~N}}\right|$, and $\left|\mathbf{F}_{i, \mathrm{E}}\right|$, are evaluated based on appropriately modified versions of Eq. (5). The functions that define the border locations $\left(f_{\mathrm{S}}, f_{\mathrm{W}}, f_{\mathrm{E}}, f_{\mathrm{N}}\right)$ are known to all actors.

The destination locations of actors are updated in discrete time steps. It was assumed for the proposed method that at each time step the destination location of $i$-th actor is shifted by a vector, which has the same direction as the resultant force $\mathbf{F}_{i}$ and length is proportional to the magnitude of this force. Thus, the updated destination location for $i$-th actor is given by the formula:

$$
\mathbf{L}_{i}^{+}=\mathbf{L}_{i}^{-}+\min \left(\alpha \cdot\left|\mathbf{F}_{i}\right|, v_{\max } \cdot \Delta t\right),
$$

where $\mathbf{L}_{i}^{-}$denotes the destination location of actor $i$ at previous time step, $\alpha$ is a scaling factor, $v_{\max }$ is maximum actor speed in meters per second, and $\Delta t$ is time step of the algorithm in seconds. It was further assumed that $\alpha=1$.

When using the proposed method, actors move until stable destination locations are found at which the repulsive forces compensate each other. If the virtual charge $q_{i}$ has the same value for each actor then the stable destination locations of actors are almost equally 
spaced. Figure 4 shows an example of the stable locations for 50 actors with equal charges in a square monitored area.

The uniform spatial distribution of actors is not effective if targets are detected more frequently in some regions of the monitored area. In such regions, where more targets have to be captured, the distances between actors should be reduced. Shorter distances between actors can be obtained by decreasing the virtual charges. Therefore in the proposed method, the actor's charge decreases with growing workload of the actor. Such effect is obtained by using the following formula to calculate current charge of $i$-th actor:

$$
q_{i}=q_{A} \cdot\left(1-\hat{w}_{i}\right)^{e},
$$

where $q_{A}$ is an initial actor's charge (equal for all actors), $\hat{w}_{i}$ denotes recent workload of $i$ th actor, and the exponent $e$ is a parameter of the algorithm. The recent workload $\hat{w}_{i}$ is estimated by using the exponential forgetting approach [25]. According to this approach, the following update operation is performed at each time step:

$$
\hat{w}_{i}=(1-\beta) \cdot \hat{w}_{i}^{-}+\beta \cdot w_{i},
$$

where $1 / \beta$ is the time constant of the forgetting process, $\hat{w}_{i}^{-}$is the recent workload of $i$-th actor estimated at previous time step, and $w_{i}$ denotes current workload of $i$-th actor $\left(w_{i}=0\right.$ if the actor is idle and $w_{i}=1$ if the actor is busy, i.e., moves toward a selected target). It is important to note that $\hat{w}_{i}$ can take values between 0 and 1 .

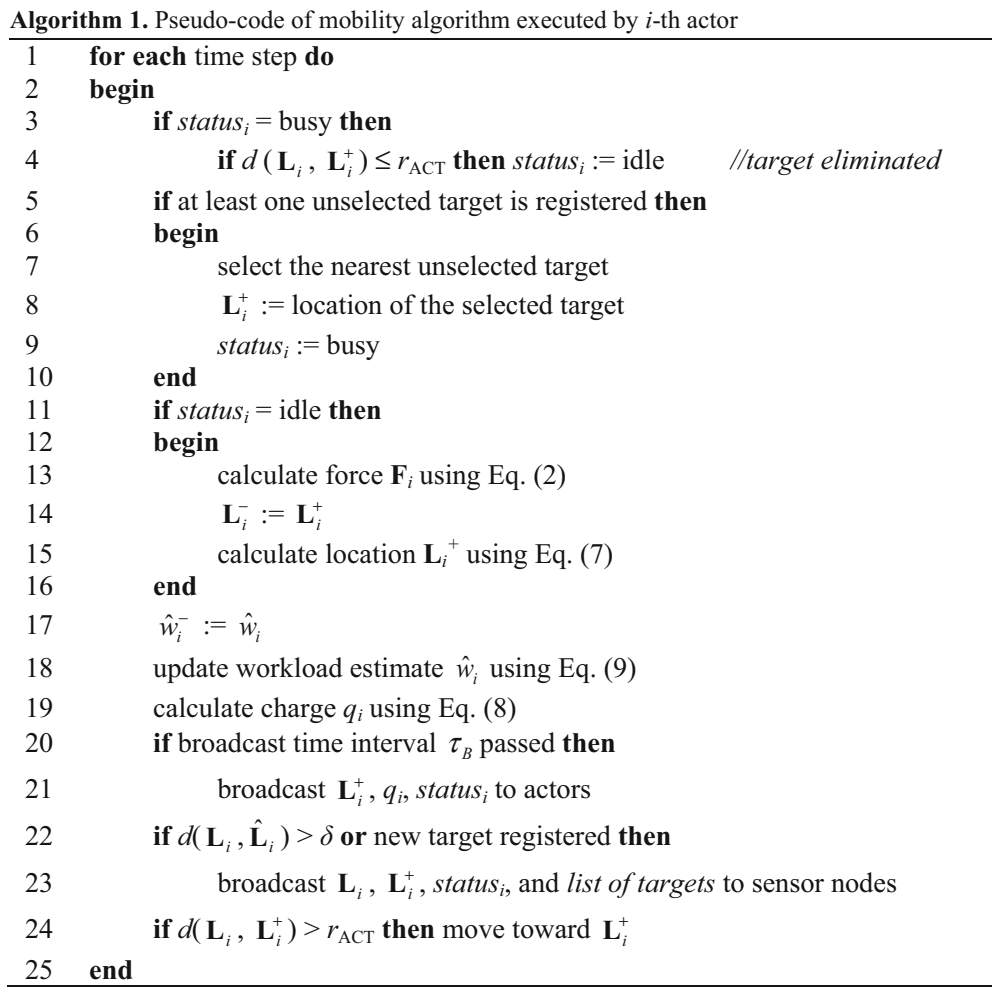




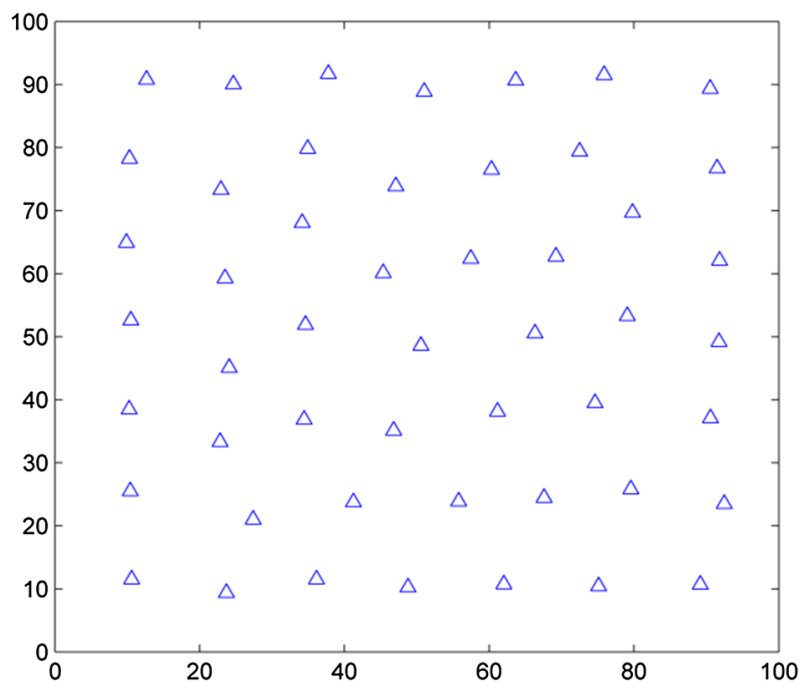

Fig. 4 Stable actor locations (triangles) in square area, no target detected

The virtual electrostatic forces govern movement of an actor only if no target was reported to the actor. When an actor receives information about locations of detected targets from sensor nodes then it moves toward the nearest target. A target is eliminated if it is within acting range of an actor. Details of the operations executed by actors are presented in Algorithm 1.

The sensor-actor communication is based on geographical routing [26]. Hence, the sensor nodes need the information about actual locations of actors. In this study, an energyaware location management scheme is applied, which includes location updating and location prediction operations [10]. According to this scheme, sensor nodes predict locations of actors based on previously received updates by using Kalman filter. An actor broadcasts updates in its communication range if its actual location $\mathbf{L}_{i}$ is far from location $\hat{\mathbf{L}}_{i}$ predicted by the sensor nodes. Additionally, the broadcasts are used as acknowledgements to handle communication failures. Thus, actors broadcast the updates after receiving a message about new detected target. Each update includes actor's actual position, destination position, status, and list of registered targets.

A sensor node, which detects a new target, reports the target location to a selected actor. Two actor selection conditions are considered in this study. According to the first actor selection condition, a sensor node reports its reading to the nearest known actor. The rationale behind this condition is that the nearest actor is expected to capture the target in shortest time and the additional benefit is that the transmission requires minimum hop count. The second actor selection condition aims at balancing the actors' workload. When using this condition, a sensor node selects the actor with minimum number of targets registered in the target list. If more actors have the same minimum number of registered targets, then the distance to target is also taken into account to select one of them.

After transmitting target coordinates, the sensor node waits for acknowledgement (broadcast) from the actor. If the acknowledgement is not received, then the coordinates are retransmitted at the next time step. The source sensor node executes retransmissions until the acknowledgement is delivered or a limit of retransmissions is reached. 


\section{Experiments}

Simulation experiments were performed to compare performance of the proposed actors' mobility control method with state-of-the-art approaches. The comparison was made by taking into account two criteria: mean time from target detection to target elimination, and mean distance covered by one actor during simulation period.

\subsection{Simulation Setup}

The simulations were conducted for six scenarios that correspond to different spatial distributions of targets and different numbers of targets generated during simulation period. Settings of the simulation scenarios are presented in Table 1. Three spatial distributions of targets are considered. In case of the uniform distribution, the probability of target occurrence is equal for all locations in the monitored area. The non-uniform distributions of target localization (single Gaussian and double Gaussian) are presented in Fig. 5. During simulation, targets are generated in time steps of one second. The simulation scenarios involve two cases described as low and high number of targets generated at one time step. These two cases are defined by the probability distributions shown in Fig. 6.

Table 2 includes parameters of the WSAN model, which was used in the simulation experiments to evaluate performance of the compared mobility control algorithms. This model was implemented in Matlab. The sensor nodes are deployed in randomized grid topology. Initially, a square grid topology is created and then the sensor nodes are distracted randomly from their initial position. Implementation of the energy model is the same as in the work by Zeng et al. [12]. This simple model assumes that during data transmission both the transmitting and the receiving nodes consume $50 \mathrm{~nJ}$ of energy per bit.

Table 1 Simulation scenarios

\begin{tabular}{lll}
\hline Scenario & Targets distribution & Targets number \\
\hline UL & Uniform & Low \\
UH & Uniform & High \\
SGL & Single Gaussian & Low \\
SGH & Single Gaussian & High \\
DGL & Double Gaussian & Low \\
DGL & Double Gaussian & High \\
\hline
\end{tabular}
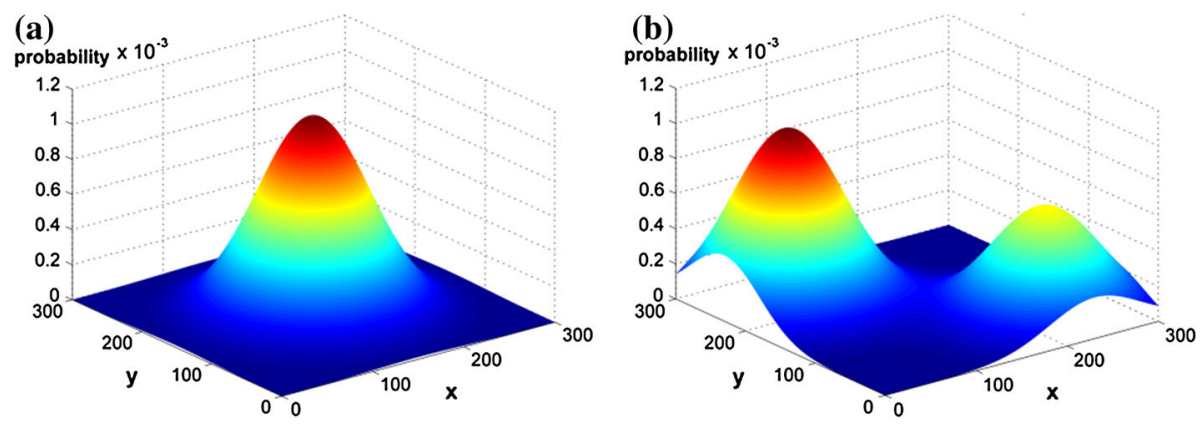

Fig. 5 Probability distributions of target localization: a single Gaussian (simulation scenarios SGL and $\mathrm{SGH}$ ), b double Gaussian (simulation scenarios DGL and DGH) 

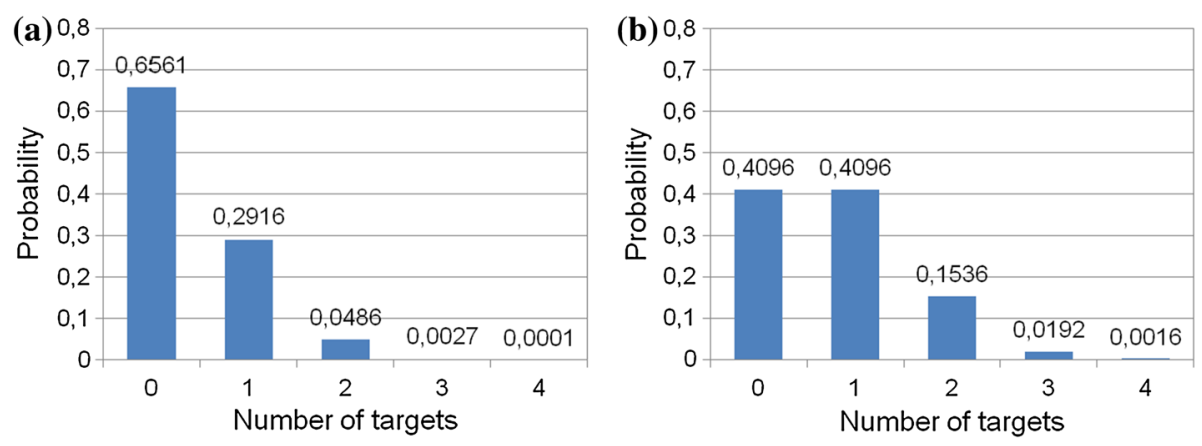

Fig. 6 Probability of creating a given number of targets at one time step: a low target intensity (simulation scenarios UL, SGL and DGL), b high target intensity (simulation scenarios UH, SGH and DGH)

Table 2 Parameters of WSAN model

\begin{tabular}{ll}
\hline Parameter & Value \\
\hline Monitored area & $300 \mathrm{~m} \times 300 \mathrm{~m}$ \\
Number of sensor nodes & 841 \\
Number of actors & 16 \\
Transmission range of sensor node & $15 \mathrm{~m}$ \\
Sensing range of sensor node & $15 \mathrm{~m}$ \\
Transmission range of actor & $60 \mathrm{~m}$ \\
Acting range of actor & $5 \mathrm{~m}$ \\
Maximum speed of actor & $2 \mathrm{~m} / \mathrm{s}$ \\
Bandwidth & $250 \mathrm{kbit} / \mathrm{s}$ \\
Packet size & $56 \mathrm{bytes}$ \\
Packet error rate & $1 \%$ \\
Broadcast time interval & $10 \mathrm{~s}$ \\
Sensor initial energy & $1 \mathrm{~J}$ \\
Sensor trans/recv power & $50 \mathrm{~nJ} / \mathrm{bit}$ \\
\hline
\end{tabular}

\subsection{Compared Algorithms}

In this study, the performance is analysed of eight mobility control algorithms that are listed in Table 3. The differences between these algorithms lie in the applied actor deployment method, and the condition of actor selection.

Algorithms EVN, EVW, ECN, and ECW are based on the proposed method, which is discussed in Sect. 3. However, in case of algorithms ECN and ECW, the proposed method is used without the procedure for modification of actor's charge according to recent actor's workload (Eq. 8). It means that for these two algorithms the virtual charges of actors are constant $\left(q_{i}=q_{\mathrm{A}}\right)$, equal to the initial value.

The last four algorithms in Table 3 utilize state-of-the-art approaches for actor deployment. According to the uniform deployment approach [15], a default initial location is determined for each actor to create an ideal square grid topology, which ensures full coverage of the monitored area. In this scheme, an actor which does not have information 
Table 3 Compared algorithms

\begin{tabular}{lll}
\hline Algorithm & Actor deployment & Actor selection \\
\hline EVN & Electrostatic, variable charge & Nearest distance to target \\
EVW & Electrostatic, variable charge & Lowest workload \\
ECN & Electrostatic, constant charge & Nearest distance to target \\
ECW & Electrostatic, constant charge & Lowest workload \\
UN & Uniform & Nearest distance to target \\
UW & Uniform & Lowest workload \\
RN & Random & Nearest distance to target \\
RW & Random & Lowest workload \\
\hline
\end{tabular}

about target locations always moves toward its default location. The random deployment scheme means that the uniform probability distribution is used to determine initial actors' locations. Each randomly deployed actor moves only if it possesses information about target location and remains static in opposite situation.

The examined algorithms combine the above methods of actor deployment with two different conditions that are used by sensor node for selection of an actor to which it reports a detected target. As discussed earlier in Sect. 3, the sensor node selects the nearest actor or the actor with the lowest workload (the lowest number of registered targets).

For all considered mobility algorithms, the decisions about actors' movements are taken in discrete time steps of one second.

\subsection{Experimental Results}

The experimental results discussed in this section were obtained from 20 simulation runs for each algorithm and scenario. The simulation run is finished when the number of eliminated targets reaches 1000 .

Initial experiments were performed to find effective settings of the algorithm parameters, i.e., the initial value of actor's charge $q_{\mathrm{A}}$, the forgetting parameter $\beta$ which is used for estimation of recent actors' workload, and the parameter $e$ of the charge function (8). The simulations were conducted for: $q_{\mathrm{A}}$ between 10 and 100, $\beta$ between 0 and 1 , and $e$ between 1 and 5. It was assumed that the virtual charge of borders $q_{\mathrm{B}}$ is equal to the initial actor's charge $q_{\mathrm{A}}$. In most of the simulation scenarios, the minimum target elimination time and the minimum distance covered by actor were observed for $q_{\mathrm{A}}=60, \beta=0.1$, and $e=3$. Therefore, these parameter settings were selected for further experiments.

The simulation results obtained for particular scenarios are shown in Figs. 7, 8, 9, 10, 11 and 12. The charts in those figures compare the mean values of target elimination time and distance covered by one actor during simulation period for all examined algorithms.

In case of the scenarios with uniformly distributed targets (UL-Fig. 7 and UHFig. 8) a low target elimination time is obtained for the mobility algorithms UN and UW that assume the ideal, uniformly distributed grid of actors. The short target elimination time can be achieved by these state-of-the-art algorithms because both the targets distribution and the actors' distribution are uniform. It should be noted that such assumptions are rarely met perfectly in practice. However, in these scenarios it can be observed that the proposed self-organizing algorithms reach the target elimination time obtained for the idealized uniform actors' deployment. When considering the scenario with high number of targets 

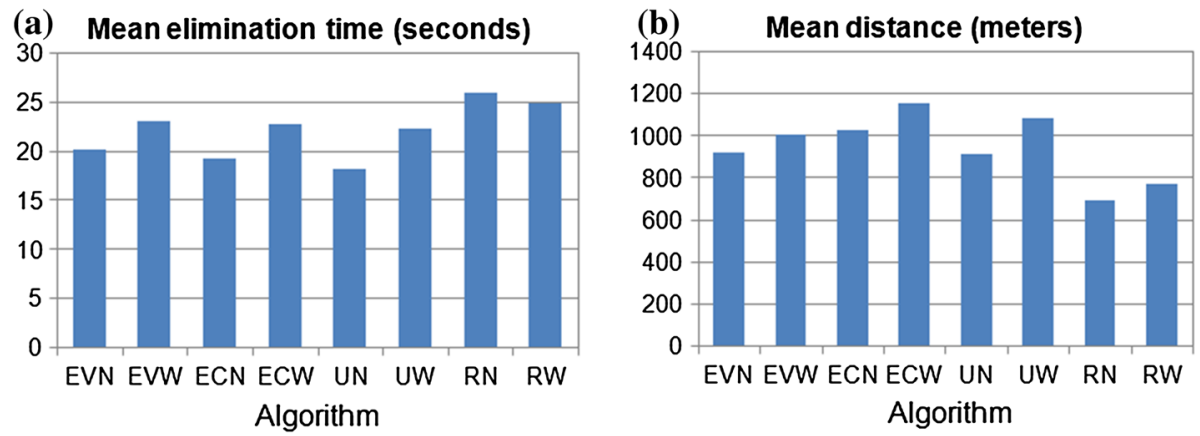

Fig. 7 Simulation results for UL scenario: a mean time between target detection and elimination, b mean distance covered by one actor during simulation period
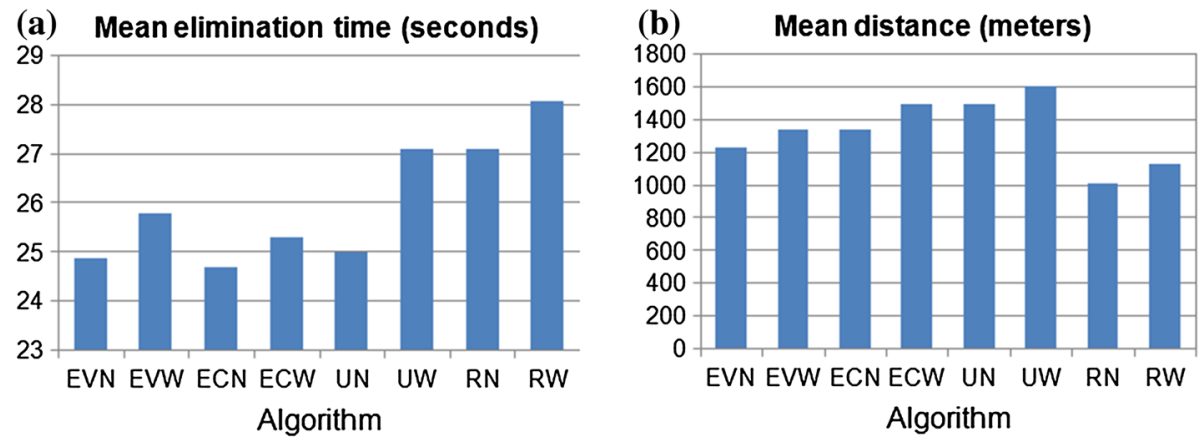

Fig. 8 Simulation results for UH scenario: a mean time between target detection and elimination, b mean distance covered by one actor during simulation period
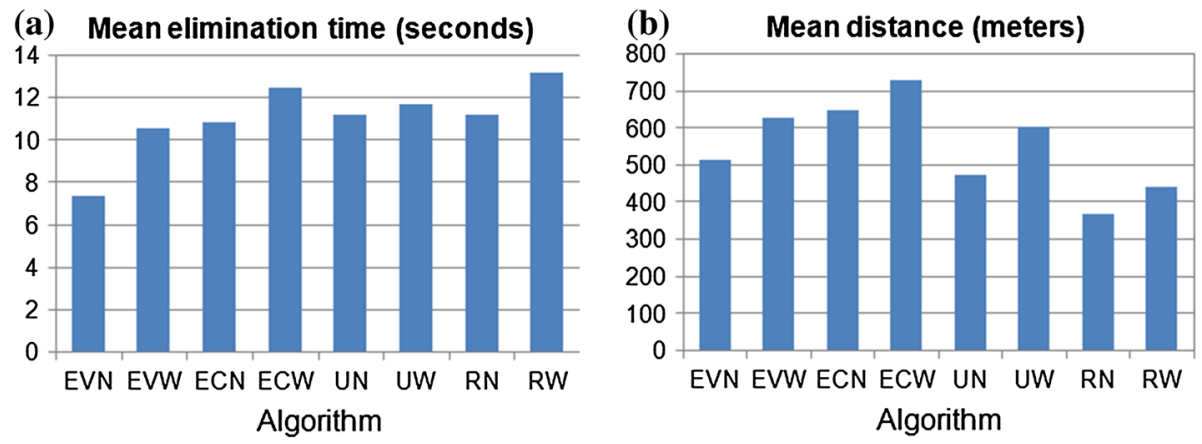

Fig. 9 Simulation results for SGL scenario: a mean time between target detection and elimination, $\mathbf{b}$ mean distance covered by one actor during simulation period

(Fig. 8a), the shortest elimination time was observed for the proposed ECN algorithm with constant charges as it tends to distribute the actors uniformly and adapts to local variations of targets density. The longest elimination time was obtained for the random actors' deployment (algorithms RN and RW). Figures $7 \mathrm{~b}$ and $8 \mathrm{~b}$ show that in both scenarios with 

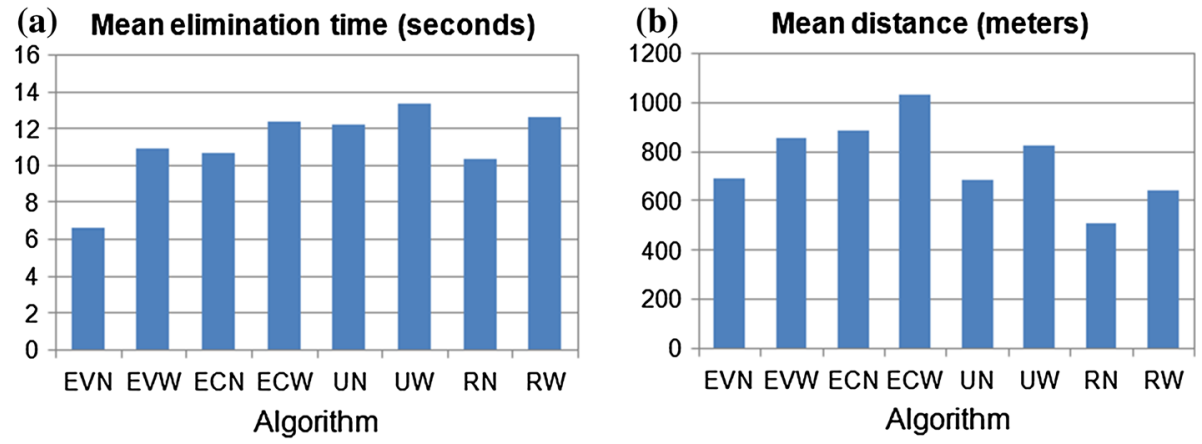

Fig. 10 Simulation results for SGH scenario: a mean time between target detection and elimination, b mean distance covered by one actor during simulation period
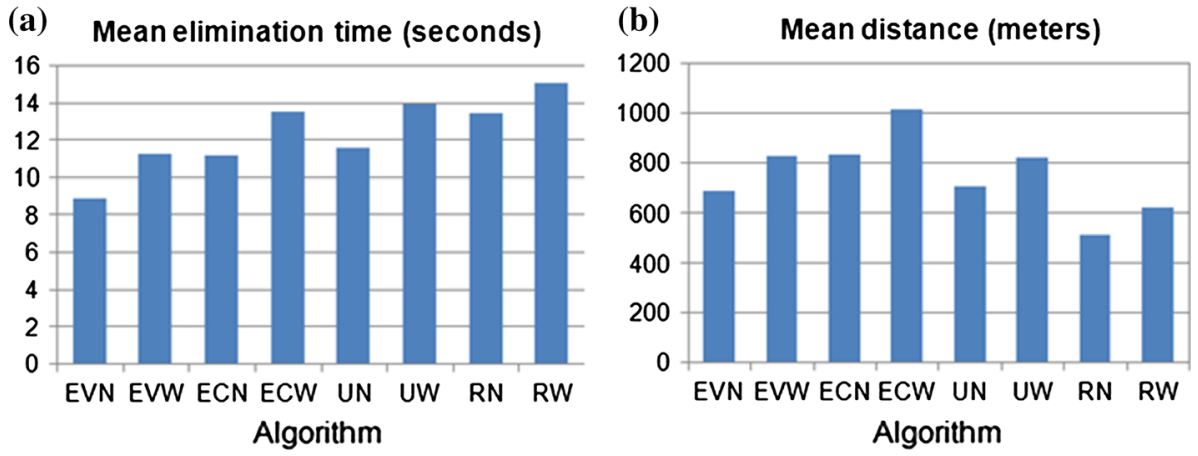

Fig. 11 Simulation results for DGL scenario: a mean time between target detection and elimination, b mean distance covered by one actor during simulation period
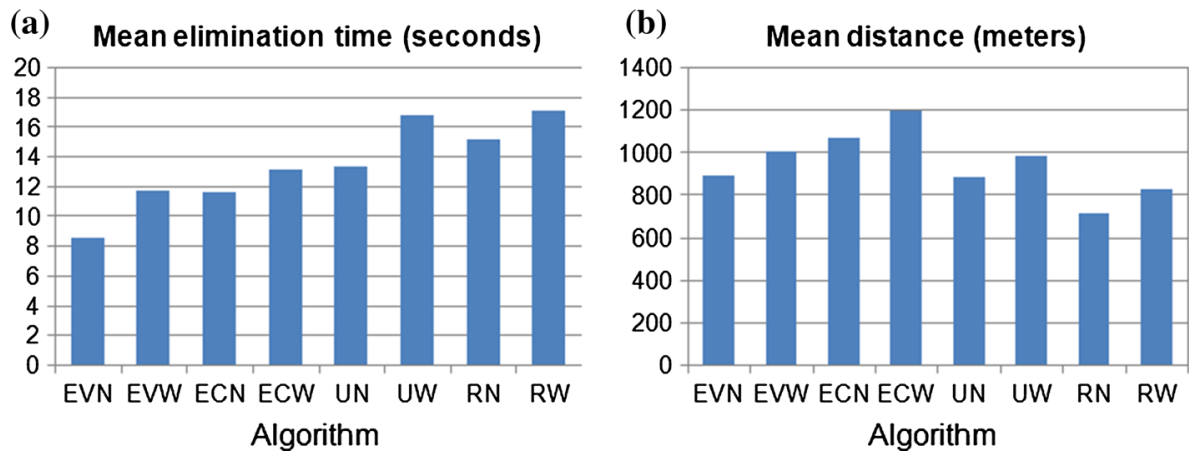

Fig. 12 Simulation results for DGH scenario: a mean time between target detection and elimination, b mean distance covered by one actor during simulation period

the uniform target distribution, the shortest mean distance covered by actor was obtained for RN and RW algorithms. For those algorithms actors do not move until they receive information about target location. This approach ensures short distances covered by actor for all scenarios but require a long time to eliminate the targets. 
The benefits of introducing variable virtual charges of actors for the proposed approach are especially visible in the simulation scenarios with Gaussian distributions of target localization. Figures 9 and 10 show the results obtained for the single Gaussian distribution, which causes a high density of targets in centre of the monitored area. In this scenario both the uniform and the random actors' deployment do not match the targets distribution. Thus, the results obtained for the state-of-the-art algorithms (UN, UW, RN, and RW) are evidently worse in comparison with those of EVN algorithm, which significantly reduces the target elimination time. The reason of this improvement is the ability of adapting the actors' deployment to the targets distribution. It should be noted that the results of the algorithm with constant actors' charges (ECN) are not as good as those of EVN, which updates the charges according to recent actor's workload. The mean distance covered by actor in case of EVN is not significantly longer than the distance obtained for the state-ofthe-art approach based on uniform actors' deployment (UN algorithm). The selection of actor with minimum workload (EVW, ECW) decreases the performance of the proposed approach.

The simulation results for DGL and DGH scenarios (Figs. 11, 12) confirm that the EVN algorithm, which is based on the proposed method, has the ability to adapt the actors' deployment to more complex target distributions than those considered in previous scenarios. In this case the double Gaussian distribution is used for targets localization. Thus, there are two regions in the monitored area, where the targets are detected more frequently. For both low and high number of targets, the EVN algorithm outperforms the remaining algorithms in terms of the target elimination time (Figs. 11a, 12a). The distance covered by actor for EVN algorithm is at the same level as that observed for UN algorithm (Figs. 11b, $12 \mathrm{~b})$. When the high number of targets is generated then all the algorithms that are based on the proposed method (EVN, EVW, ECN, and ECW) allow a lower elimination time to be obtained in comparison with the state-of-the-art approaches (Fig. 12a).

Figure 13 illustrates actors' deployments in SGL simulation scenario for different algorithms. In this figure the crosses represent targets that correspond to current destinations of selected actors, and circles show new targets, which will be eliminated subsequently. It can be observed in these examples that the proposed method adapts the actor deployment to the single Gaussian distribution of targets (Fig. 13a, b). The actors' locations are closer to the new targets thus the shorter elimination time can be obtained. In case of EVN algorithm, actors are localised closer to the centre of the monitored area because the virtual repulsive forces are decreased for the busy actors. When using the state-of-theart algorithms (Fig. 13c, d), the actors localized close to the borders of the monitored area are idle most of the time.

Further simulation experiments were conducted to take into account failures of actors. It was assumed that probability of failure equals 0.004 at each time step for every actor. After failure, the actor is removed from the simulation. Thus, 4 actors are removed on average during the simulation period of 1000 time steps (seconds). Results of the simulations for the UL, SGL, and DGL scenarios with actor failures are presented in Figs. 14, 15 and 16. These results reveal robustness of the proposed approach. The proposed method allows the actors to be appropriately repositioned after failure, without significant negative effect on the performance. Thus, the algorithms that are based on the proposed method achieve shorter target elimination time than the state-of-the-art approaches. It should be also noted that in case of scenarios with actor failures the mean distance covered by actor for the proposed algorithms (EVN, EVW, ECN) is at the same low level as for the algorithms with random actors deployment $(\mathrm{RN}, \mathrm{RW})$. 
(a)

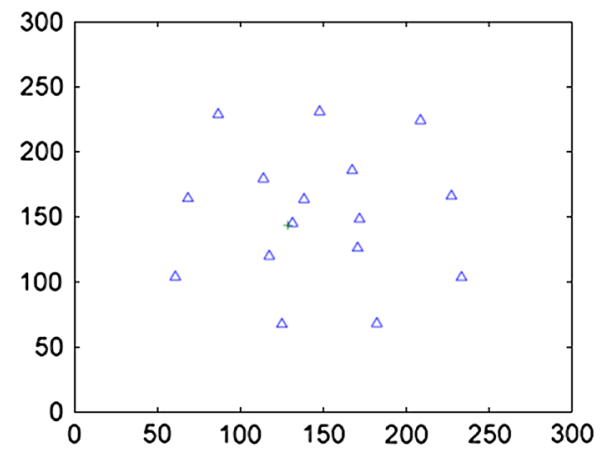

(c)

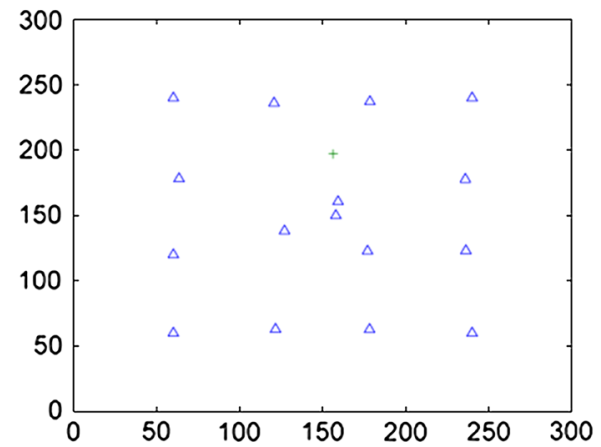

(b)

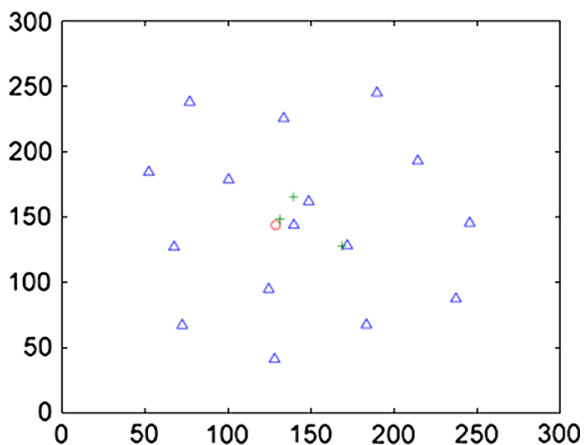

(d)

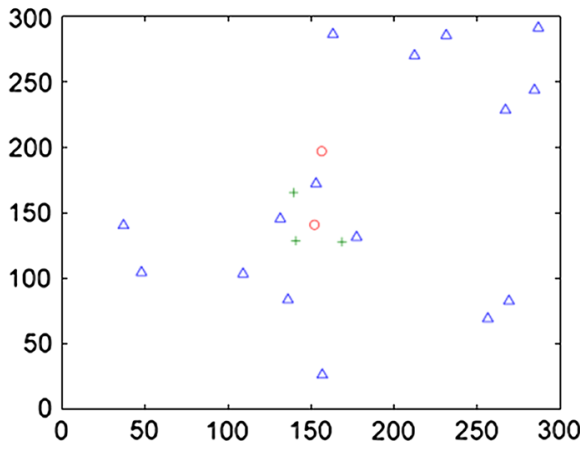

Fig. 13 Snapshots of WASNS deployment in SGL scenario for the compared algorithms: a EVN, b ECN, c UN, $\mathbf{d}$ RN (actors are represented by triangles, targets are shown as circles and crosses)
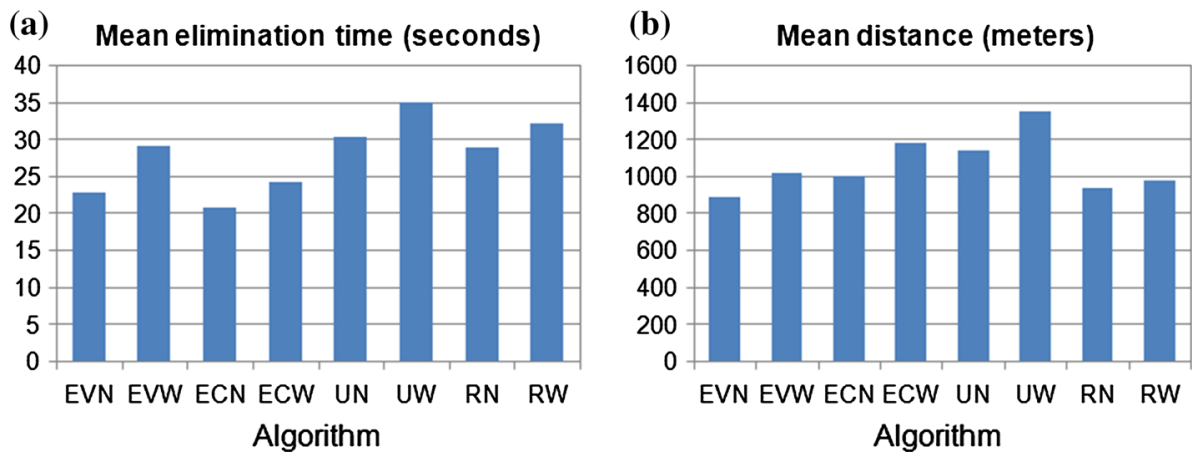

Fig. 14 Simulation results for UL scenario with actor failures: a mean time between target detection and elimination, $\mathbf{b}$ mean distance covered by one actor during simulation period

Figure 17 shows examples of actors' deployments during simulation of SGL scenario with failures. In these examples it can be observed that the introduced method suitably relocates the actors after failure to ensure coverage of the central region, where the targets are detected more frequently (Fig. 17a, b). The state-of-the-art algorithms (Fig. 17c, d) leave some parts of this area uncovered. 

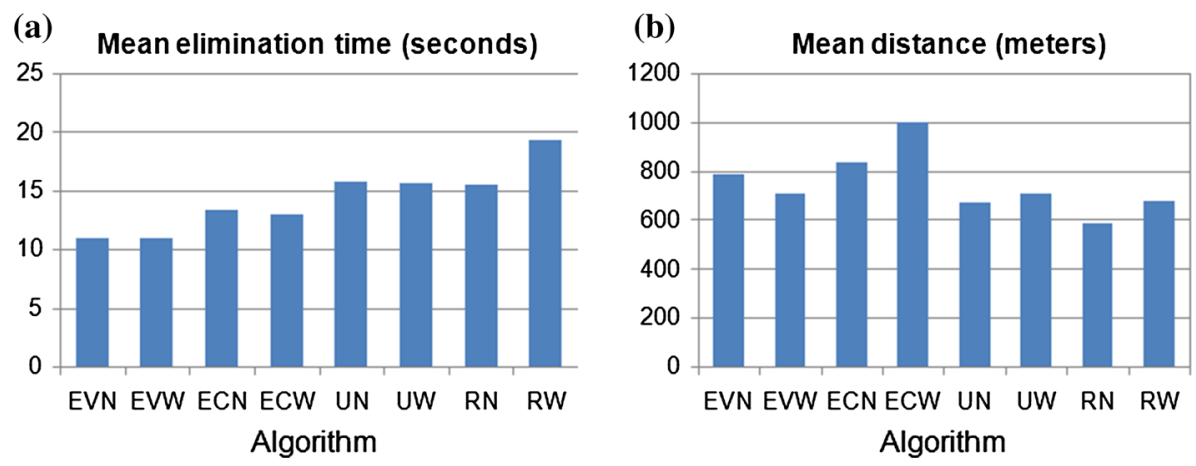

Fig. 15 Simulation results for SGL scenario with actor failures: a mean time between target detection and elimination, $\mathbf{b}$ mean distance covered by one actor during simulation period
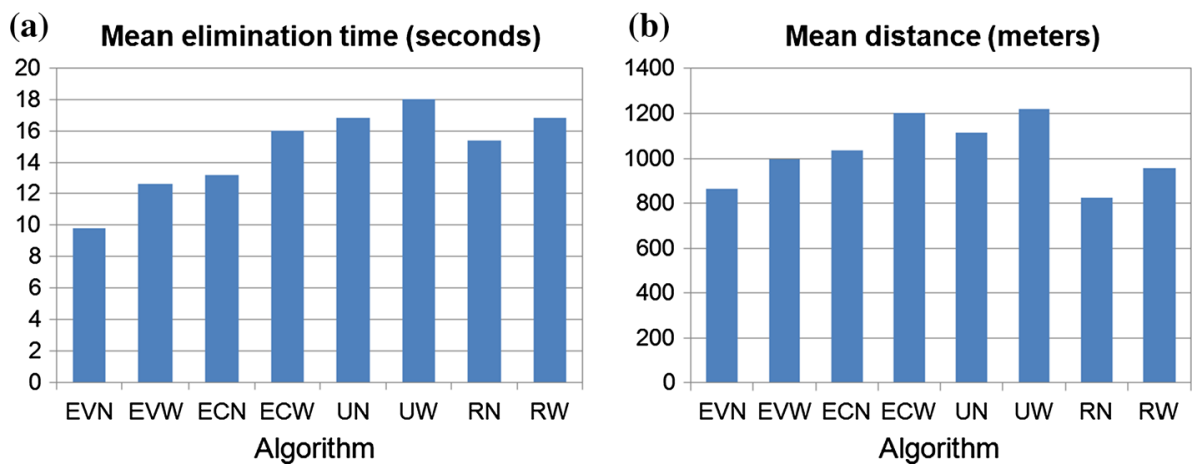

Fig. 16 Simulation results for DGL scenario with actor failures: a mean time between target detection and elimination, $\mathbf{b}$ mean distance covered by one actor during simulation period

Energy consumption of sensor nodes is an important factor for the effectiveness of WSAN applications. Thus, residual sensor energy was evaluated during the simulation experiments. Results of this evaluation are presented in Fig. 18. These results were collected after $5000 \mathrm{~s}$ of the simulation and averaged for all considered scenarios. The lowest energy consumption and minimum standard deviation of the residual energy was observed for EVN algorithm. The EVN algorithm uses the proposed mobility control method, which ensures a relatively low distance between actors and detected targets, especially for the scenarios with non-uniform target distribution. Thus, lower number of sensor nodes is involved in relaying data to the actors and the mean energy consumption is reduced. In case of the uniform targets distribution (scenarios UL and UH) the energy consumption for the proposed method is at the same level as for the state-of-the-art approaches. For remaining scenarios, the proposed method reduces the energy consumption. It should be also noted that the residual energy is more evenly dispersed across the sensor nodes in the network.

According to the presented results, it can be concluded that the proposed approach enables more effective organization of the actors' mobility than the state-of-the-art methods. In case of the uniform target distribution with low number of targets, the proposed approach allows the actors to eliminate the detected targets as fast as in case of the 
(a)

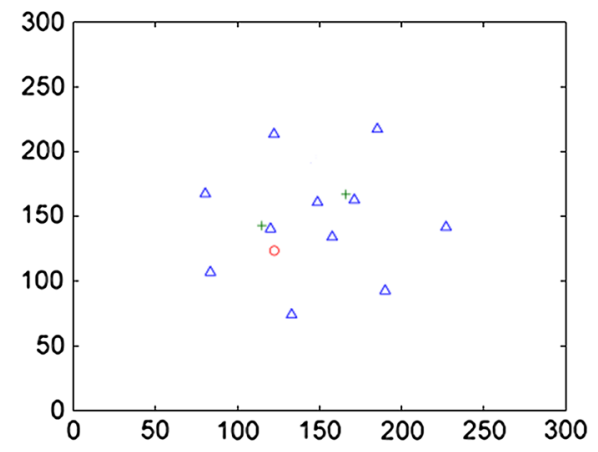

(c)

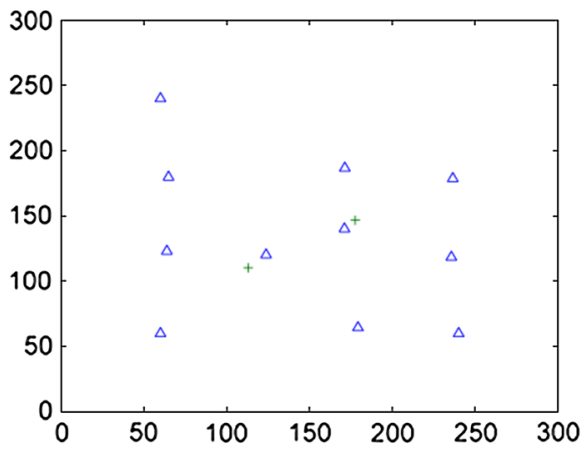

(b)

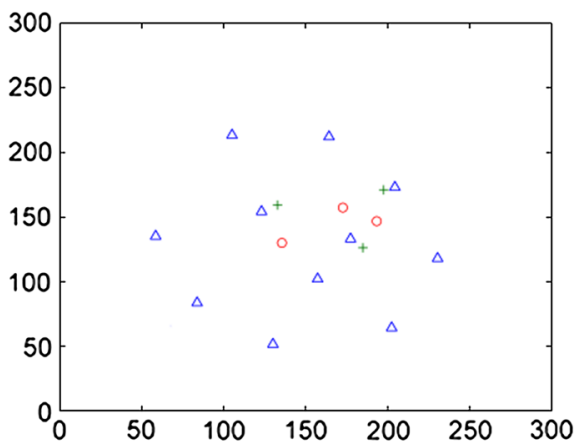

(d)

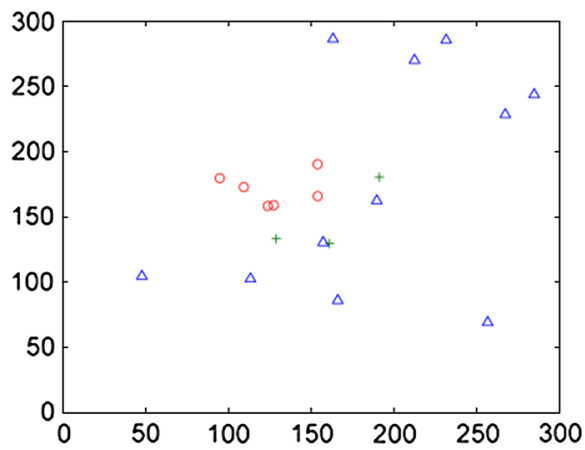

Fig. 17 Snapshots of WASNS deployment in SGL scenario with actor failures for the compared algorithms: a EVN, b ECN, c UN, d RN (actors are represented by triangles, targets are shown as circles and crosses)
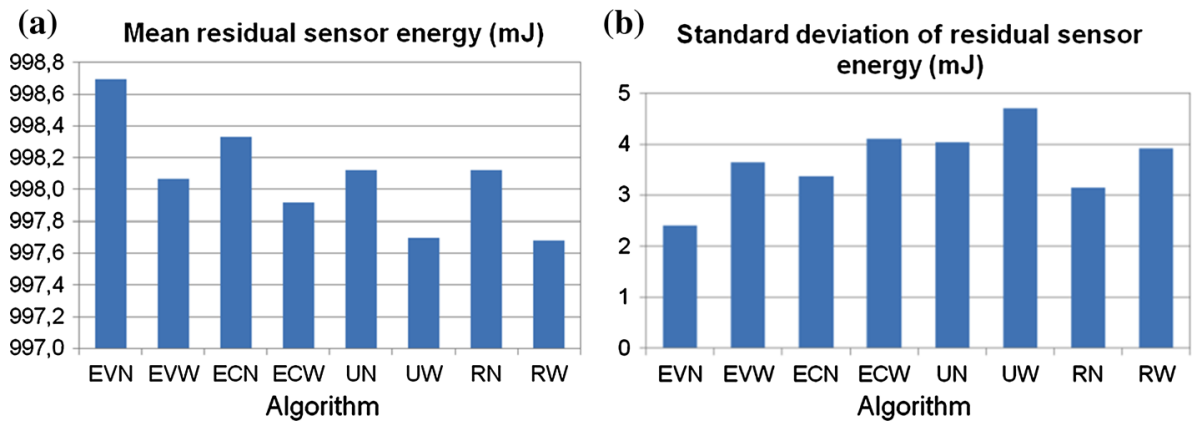

Fig. 18 Residual energy of sensor nodes averaged for all scenarios: a mean value of residual sensor energy, b standard deviation of residual sensor energy

idealized square grid deployment. For all remaining scenarios, a considerably shorter time of target elimination can be obtained by using the proposed approach. The distance covered by one actor during simulation period for the proposed approach is kept at the low level for the realistic scenarios that take into account actor failures. The best results were obtained for EVN algorithm, which is based on the proposed virtual electrostatic 
interactions with variable actors' charges. In general, the proposed algorithms with variable virtual actors' charges (EVN, EVW) obtain a better performance that their counterparts with constant charges (ECN, ECW). The introduction of variable actors' charges improves the control performance especially for non-uniform target distributions. When comparing the proposed algorithms that use the selection of nearest actor (EVN, ECN) with those that implement the selection of actor with lowest workload (EVW, ECW), it is evident that the proposed approach performs better if the nearest actor is selected. The introduced method enables effective self-organized actors' relocation in case of actor failure. Thus the superior performance can be achieved by this method also in case when some actors are out of order.

\section{Conclusions}

New surveillance applications of WSANs require appropriate algorithms to control the mobility of actors. The mobility control algorithms should enable a self-organizing deployment of actors in the monitored area and ensure a fast access of actors to detected targets. In this paper a mobility control method is introduced based on virtual electrostatic interactions inspired by Coulomb's law from physics. The objective of this method is to enable effective relocation of actors that have to reach targets detected by sensor nodes in minimum time.

The virtual forces allow the actors' deployment to be dynamically adapted to local probability of target detection. The adaptation ability was obtained for the proposed method by introducing variable virtual electrostatic charges of actors. The virtual charge of an actor is updated as a function of recent actor's workload. Exponential forgetting algorithm is applied to estimate the workload of each actor in some recent time period. The proposed definition of virtual electrostatic charges leads to reduced distances between actors in regions, where targets are detected more frequently. Thus, the resulting spatial distribution of actors is more effective than the uniform one, and the targets can be eliminated in shorter time. In case of actor failure, the proposed method allows the remaining active actors to be appropriately repositioned. Moreover, the introduced approach is beneficial owing to low consumption of sensor nodes energy. Main advantages of the proposed self-organizing mobility control are robustness, scalability, flexibility, adaptivity, and low computational cost.

In contrast with the state-of-the-art methods, the proposed method does not require any additional energy-expensive communication with sensor nodes. The virtual forces and charges are determined on the basis of data transmitted only between neighbouring actors. They enable appropriate relocation of actors prior to target detection. This method is effective also in case when the locations of targets cannot be accurately predicted.

Effectiveness of the proposed data mobility control method for WSAN was verified in simulation experiments. The experimental results show that proposed method enables more effective organization of the actors' mobility than the state-of-the-art approaches based on uniform and random deployment. In comparison with the state-of-the-art solutions, the proposed method has enabled considerable reduction in mean target elimination time. It ensures a relatively low mean distance that has to be covered by actors during targets chasing. The benefits of introducing variable virtual charges of actors for the proposed method are especially visible in case of non-uniform distributions of target localization. 
Robustness of the proposed method was demonstrated in simulation scenarios that take into account actor failures.

An interesting topic for future works is to investigate applicability of the proposed method for surveillance tasks with mobile targets in complex scenarios with obstacles. Another direction for future research is experimental evaluation of the proposed approach in more advanced simulators that include detailed models of the physical layer and the wireless channel. It is also planned to verify robustness, scalability, flexibility, adaptivity and evaluate computational cost of the introduced approach in a real-world test bed. The real-world experiments will be conducted using Zigbee or Bluetooth Low Energy mesh communication. The actors will be implemented by means of mobile platforms (robots) managed by system on chip and supporting the aforementioned transmission standards.

Open Access This article is distributed under the terms of the Creative Commons Attribution 4.0 International License (http://creativecommons.org/licenses/by/4.0/), which permits unrestricted use, distribution, and reproduction in any medium, provided you give appropriate credit to the original author(s) and the source, provide a link to the Creative Commons license, and indicate if changes were made.

\section{References}

1. Ranga, V., Dave, M., \& Verma, A. K. (2014). A hybrid timer based single node failure recovery approach for WSANs. Wireless Personal Communications, 77(3), 2155-2182.

2. Liu, B. H., Tang, Y. J., Yu, C. W., \& Tsai, M. J. (2015). Greedy algorithms for actor redeployment in wireless sensor-actor networks. Wireless Networks, 21(2), 431-442.

3. Kamali, M., Laibinis, L., Petre, L., \& Sere, K. (2014). Formal development of wireless sensor-actor networks. Science of Computer Programming, 80, 25-49.

4. Ranga, V., Dave, M., \& Verma, A. K. (2013). Network partitioning recovery mechanisms in WSANs: A survey. Wireless Personal Communications, 72(2), 857-917.

5. Khamis, A., \& ElGindy, A. (2012). Minefield mapping using cooperative multirobot systems. Journal of Robotics, 2012(698046), 1-7.

6. Vedantham, R., Zhuang, Z. \& Sivakumar, R. (2006). Mutual exclusion in wireless sensor and actor networks. In: 3rd annual IEEE communications society on sensor and ad hoc communications and networks SECON'O6 (Vol. 1, pp. 346-355).

7. Kułakowski, P., Calle, E., \& Marzo, J. L. (2013). Performance study of wireless sensor and actuator networks in forest fire scenarios. International Journal of Communication Systems, 26(4), 515-529.

8. Gaj, P., Jasperneite, J., \& Felser, M. (2013). Computer communication within industrial distributed environment-a survey. Industrial Informatics, IEEE Transactions on, 9(1), 182-189.

9. Bernas, M. (2013). WSN power conservation using mobile sink for road traffic monitoring. In A. Kwiecień, P. Gaj \& P. Stera (Eds.), Computer networks (pp. 476-484). Berlin: Springer.

10. Melodia, T., Pompili, D., \& Akyldiz, I. F. (2010). Handling mobility in wireless sensor and actor networks. IEEE Transactions on Mobile Computing, 9(2), 160-173.

11. Li, X., Santoro, N., \& Stojmenovic, I. (2009). Localized distance-sensitive service discovery in wireless sensor and actor networks. IEEE Transactions on Computers, 58(9), 1275-1288.

12. Zeng, Y., Li, D., \& Vasilakos, A. V. (2013). Real-time data report and task execution in wireless sensor and actuator networks using self-aware mobile actuators. Computer Communications, 36(9), 988-997.

13. Mezei, I., Lukic, M., Malbasa, V., \& Stojmenovic, I. (2013). Auctions and iMesh based task assignment in wireless sensor and actuator networks. Computer Communications, 36(9), 979-987.

14. Ota, K., Dong, M., Cheng, Z., Wang, J., Li, X., \& Shen, X. S. (2012). ORACLE: Mobility control in wireless sensor and actor networks. Computer Communications, 35(9), 1029-1037.

15. Płaczek, B., \& Bernas, M. (2015). Data suppression algorithms for surveillance applications of wireless sensor and actor networks. In P. Gaj, A. Kwiecień \& P. Stera (Eds.), Computer networks (pp. 23-32). Cham: Springer.

16. Spears, W. M., Spears, D. F., Hamann, J. C., \& Heil, R. (2004). Distributed, physics-based control of swarms of vehicles. Autonomous Robots, 17(2-3), 137-162.

17. Younis, M., Senturk, I. F., Akkaya, K., Lee, S., \& Senel, F. (2014). Topology management techniques for tolerating node failures in wireless sensor networks: A survey. Computer Networks, 58, 254-283. 
18. Xiaoping, R., Zixing, C., Zhao, L., \& Wuyi, W. (2012). Performance analysis of virtual force models in node deployment algorithm of WSN. In G. Lee (Ed.), Advances in intelligent systems (pp. 1-9). Berlin: Springer.

19. Heo, N., \& Varshney, P. K. (2005). Energy-efficient deployment of intelligent mobile sensor networks. IEEE Transactions on Systems, Man, and Cybernetics Part A: Systems and Humans, 35(1), 78-92.

20. Senturk, I. F., Akkaya, K., \& Yilmaz, S. (2012). Distributed relay node positioning for connectivity restoration in partitioned wireless sensor networks. In IEEE symposium on computers and communications (ISCC) (pp. 301-306).

21. Imran, M., Younis, M., Said, A. M., \& Hasbullah, H. (2010). Volunteer-instigated connectivity restoration algorithm for wireless sensor and actor networks. In Wireless communications, international conference on networking and information security (WCNIS) (pp. 679-683).

22. Joshi, Y. K., \& Younis, M. (2012). Autonomous recovery from multi-node failure in wireless sensor network. In Global communications conference (GLOBECOM) (pp. 652-657).

23. Garetto, M., Gribaudo, M., Chiasserini, C. F., \& Leonardi, E. (2008). Sensor deployment and relocation: A unified scheme. Journal of computer science and technology, 23(3), 400-412.

24. Akkaya, K., \& Janapala, S. (2008). Maximizing connected coverage via controlled actor relocation in wireless sensor and actor networks. Computer Networks, 52(14), 2779-2796.

25. Kulhavý, R., \& Kraus, F. J. (1996). On duality of regularized exponential and linear forgetting. Automatica, 32(10), 1403-1415.

26. Zhang, H., \& Shen, H. (2010). Energy-efficient beaconless geographic routing in wireless sensor networks. IEEE Transactions on Parallel and Distributed Systems, 21(6), 881-896.

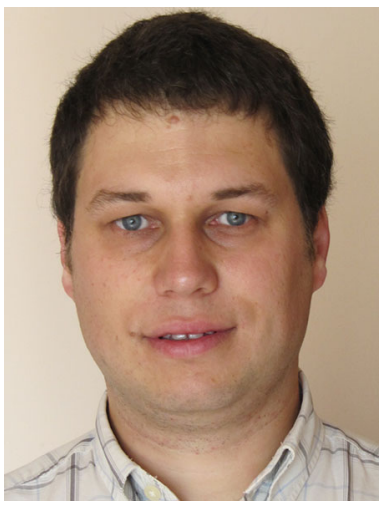

Bartłomiej Płaczek received the M.Sc. and Ph.D. degrees from Silesian University of Technology (SUT), Gliwice, Poland in 2002 and 2005, respectively. In 2016 he received the habilitation degree (D.Sc.) in computer science from SUT. From 2005 to 2013 he has been with the Faculty of Transport at SUT, while since 2013 he is an assistant professor at the Institute of Computer Science, University of Silesia, Sosnowiec, Poland. He has authored or co-authored more than seventy papers in peer-reviewed journals and conferences. He serves as a reviewer for many scientific journals, international conferences, and research founds. His current research interests include sensor networks, vehicular ad-hoc networks, intelligent transportation systems, image processing, cellular automata, and soft computing.

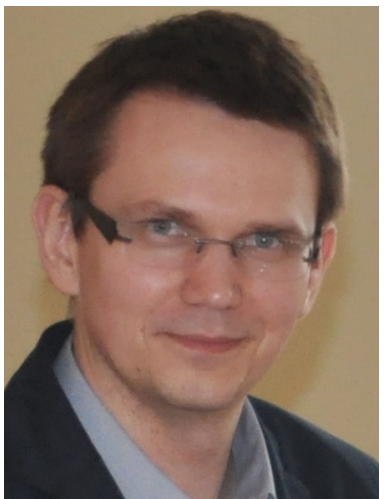

Marcin Bernaś received the M.Sc. degree from University of Silesia, Katowice, Poland in 2005 and Ph.D. degree from Silesian University of Technology in 2009. He currently works as an assistant professor at the Institute of Computer Science at University of Silesia. He is a reviewer for several renowned journals in computer science field and published over 30 scientific papers concerning wireless sensor networks, data mining, granular computing and decision support systems (classification and forecasting) for transport/medicine. 